JOCHEM KAHL

\title{
Zu den Namen spätzeitlicher Usurpatoren, Fremdherrscher, Gegen- und Lokalkönige*
}

\section{Ausgangsüberlegungen}

Daß ägyptische Könige in ihren Namen das politische Selbstverständnis ihrer Regierung in offizieller Formulierung darlegten und einen Hinweis auf ihr „Regierungsprogramm“ gaben, ist allgemein bekannt. ${ }^{1} \mathrm{Daß}$ sie diese Namen auch in der Spätzeit gezielt zur Legitimation benutzten, soll im folgenden exemplarisch gezeigt werden.

Ausgehend von der im Sonderforschungsbereich 493 entwickelten Fragestellung, ${ }^{2}$ ob und wie sich eine bestimmte Königstheologie auf die Ausübung monarchischer Herrschaft auswirkt und $o b$ und wie Ereignisse und Zwänge, mit denen monarchische Herrschaft konfrontiert wird, verändernd auf die Königstheologie einwirkten, sollen die Königsnamen von „regelkon-

* Dieser Artikel entstand im Rahmen des SFB 493 „Funktionen von Religion in antiken Gesellschaften des Vorderen Orients".

Vgl. z. B. Louise Gestermann, Kontinuität und Wandel in Politik und Verwaltung des frühen Mittleren Reiches in Ägypten (GOF IV/18; Wiesbaden 1987) 49-53 (zu den Horusnamen Mentuhoteps II.) und Rolf Gundlach, ,Weltherrscher und Weltordnung - Legitimation und Funktion des ägyptischen Königs am Beispiel Thutmosis III. und Amenophis III.", in: Rolf Gundlach - Hermann Weber (Hrsg.), Legitimation und Funktion des Herrschers: vom ägyptischen Pharao zum neuzeitlichen Diktator (Schriften der Mainzer Philosophischen Fakultätsgesellschaft 13; Stuttgart 1992) 36.

${ }^{2}$ Im SFB 493 „Funktionen von Religion in antiken Gesellschaften des Vorderen Orients" werden vier große Themenbereiche erforscht: „Religion und Regionalität", „Religion in der Krise“, „Religion und Herrschaft" sowie „Religiöse Integration und Abgrenzung“. Die Münsteraner Ägyptologie ist in der Abteilung „Religion und Herrschaft" vertreten, genauer arbeitet sie dort in der Gruppe „Thronwechsel und Usurpationen in Kleinasien, Mesopotamien, Persien, Israel und Ägypten“. formen" Thronfolgern, Usurpatoren, Fremdherrschern sowie Gegen- und Lokalkönigen hinsichtlich bestimmter legitimatorischer Aussagen miteinander verglichen werden. Denn insbesondere dort, wo Königsherrschaft prekär wird - fraglos beim Wechsel der Herrscher -, ist zu vermuten, daß Legitimationsbemühungen der Könige stattgefunden haben. Somit bietet eine Analyse der Königsnamen, die sich die neuen Machthaber zumeist bei Herrschaftsbeginn gelegentlich auch erst im Verlaufe ihrer Amtsführung - gaben (geben ließen) ${ }^{3}$, die Möglichkeit, eventuelle legitimatorische Funktionen der Namen zu erkennen.

Hierbei wird der regelhafte Fall des Thronwechsels, der in Ägypten durch Patrifiliation und/oder Designation (gelegentlich auch göttliche Erwählung) gerechtfertigt wurde, mit Spezialfällen verglichen ${ }^{4}$.

Die ägyptische Spätzeit, die in vorliegender Arbeit als der Zeitraum von der Unterwerfung Ägyptens durch Pije bis einschließlich Alexander IV. ${ }^{5}$ definiert ist, ist durch zahlreiche Fremd-

${ }^{3} \mathrm{Vgl}$. die naophore Statue des Udjahorresnet $\mathrm{Mu}-$ seo Gregoriano Egizio Vaticano Inv. Nr. 196), auf der erwähnt wird (B 13), daß Udjahorresnet die Titulatur des Kambyses mit dessen Thronnamen entworfen hat.

${ }^{4}$ Legitimation konnte der Herrscher auf verschiedene Art erlangen:

1. durch blutsmäßige Abstammung

2. durch Designation

3. durch gottesähnliche Erwählung mittels Mythos (dem König wurde beim Geburtsritual die Rolle des Gottessohnes zuteil), Orakel (das Götterbild gab bei einer Festprozession durch ein Zeichen die Wahl des Königs zu erkennen) oder Traum (dem König wurde im Traum die Königswürde verheißen)

4. durch Leistungsfähigkeit und damit verbunden dem Besitz der tatsächlichen Regierungsgewalt (z. B. Usurpation).

$$
{ }^{5} \text { Ca. } 730-306 \text { v. Chr. }
$$




\begin{tabular}{|c|c|c|c|c|c|}
\hline Dynastie & $\begin{array}{l}\text { Fremdherr- } \\
\text { scher }\end{array}$ & $\begin{array}{l}\text { Gegen-/ } \\
\text { Lokalkönige }\end{array}$ & Usurpatoren & $\begin{array}{l}\text { Befreier } \\
\text { von Fremd- } \\
\text { herrschaft }\end{array}$ & $\begin{array}{l}\text { "regelkonforme“ } \\
\text { Thronfolger }\end{array}$ \\
\hline $\begin{array}{l}\text { 25. Dyn. } \\
\text { (Kuschiten) }\end{array}$ & $\begin{array}{c}\text { Kuschiten } \\
\text { Assyrer }\end{array}$ & $\begin{array}{l}\text { Lokalkönige } \\
\text { im Delta }\end{array}$ & & & \\
\hline $\begin{array}{l}\text { 26. Dyn. } \\
\text { (Saiten) }\end{array}$ & & & Amasis & Psametik I. & $\begin{array}{c}\text { Necho II. } \\
\text { Psametik II. } \\
\text { Apries } \\
\text { Psametik III. }\end{array}$ \\
\hline $\begin{array}{l}\text { 27. Dyn. } \\
\text { (Perser) }\end{array}$ & Perser & $\begin{array}{c}\text { Pedubaste IV. } \\
\text { Inaros }\end{array}$ & & & \\
\hline $\begin{array}{l}\text { 28. Dyn. } \\
\text { (Saiten) }\end{array}$ & & & & Amyrtaios & \\
\hline $\begin{array}{l}\text { 29. Dyn. } \\
\text { (Mendesier) }\end{array}$ & & & $\begin{array}{c}\text { Nephorites I. } \\
\text { Psamuthis }\end{array}$ & & $\begin{array}{c}\text { Muthis (?) } \\
\text { Achoris (Usurpator?) } \\
\text { Nephorites II. }\end{array}$ \\
\hline $\begin{array}{l}\text { 30. Dyn. } \\
\text { (Sebennyten) }\end{array}$ & & 35 & $\begin{array}{l}\text { Nektanebis (I.) } \\
\text { Nektanebos (II.) }\end{array}$ & 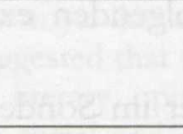 & Teos \\
\hline $\begin{array}{l}\text { 31. Dyn. } \\
\text { (Perser) }\end{array}$ & Perser & Chababasch & 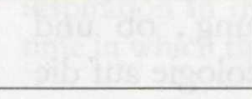 & & \\
\hline $\begin{array}{c}\text { Makedonische } \\
\text { Zeit }\end{array}$ & Argeaden & & 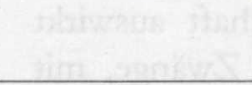 & 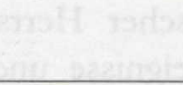 & Fingorat \\
\hline
\end{tabular}

Abb. 1. Übersicht über die Könige der ägyptischen Spätzeit, aufgeteilt nach Mechanismen ihrer Amtsübernahme

herrschaften sowie durch mehrere Dynastiewechsel und Usurpationen gekennzeichnet. Sie bietet somit ein vielversprechendes Arbeitsfeld, um zu erforschen, ob und wie Ereignisse und Zwänge, mit denen monarchische Herrschaft konfrontiert wurde, verändernd auf die Königstheologie einwirkten.

Im folgenden werden die Herrscher der Spätzeit in verschiedene Kategorien eingeteilt (s. Abb. 1), um dann den Aussagen ihrer Königsnamen eventuelle Legitimationsstrategien zu entnehmen.

Es lassen sich grob unterscheiden:

Fremdherrscher: Das sind die Könige der 25., 27. und 31. Dynastie, und das sind die Argeaden ${ }^{6}$. Stammten diese Herrscher auch aus verschiedenen Kulturkreisen, so war ihnen dennoch

${ }^{6}$ Zur Einschätzung der 25. Dynastie als Fremdherrschaft vgl. zuletzt Karl Jansen-Winkeln, „Die Fremdherrschaft in Ägypten im 1. Jahrtausend v. Chr.“, in: Or 69, 2000, 13-16. gemein, daß sie in Ägypten vor der gleichen schwierigen Situation standen, sich zu legitimieren und ihre Herrschaft anerkannt zu bekommen. Unregelmäßigkeiten in der Thronfolge dieser Dynastien bleiben unberücksichtigt, da die einzelnen Herrscher ihren indigenen Thronfolgeregeln unterlagen und nicht den ägyptischen?

Lokal-bzw. Gegenkönige: Das sind Herrscher, die im Schatten einer fremden Großmacht operierten und nur über einen Teil des Landes die Kontrolle hatten. Zur Zeit der 25. Dynastie waren dies im Delta unter anderen Gemnefchonsbak $^{8}$ und Necho I. von Sais ${ }^{9}$, zur

$\mathrm{Zu}$ der Auswahl des Königs in Kusch vgl. Angelika Lohwasser, „Die Auswahl des Königs in Kusch", in: Beiträge zur Sudanforschung 7, Wien 2000, 85-102.

${ }^{8}$ Vgl. Pierre Montet, Le lac sacré de Tanis (Paris 1966) $70-73$.

Kenneth A. Kitchen, The Third Intermediate Period in Egypt (1100-650 B.C.) (Warminster $\left.{ }^{2} 1986\right)$ 145-147. 
Zeit der 27. Dyn. waren dies Pedubaste IV. ${ }^{10}$ und Inaros ${ }^{11}$, zur Zeit der 31. Dynastie war dies Chababasch $^{12}$.

Usurpatoren: Das sind Könige, die ihre Vorgänger - zum Teil unter massiver Gewaltanwendung - absetzten und selbst die Macht ergriffen ${ }^{13}$ :

- Amasis, der nach Herodot II, 161-162 vom meuternden Heer zum König gegen Apries erhoben wurde ${ }^{14}$,

- Nephorites I. ${ }^{15}$, der Amyrtaios gefangen und hingerichtet haben soll ${ }^{16}$,

- Psamuthis, der John Ray zufolge ein Usurpator, nicht nur ein Gegenkönig zu Achoris war ${ }^{17}$,

${ }^{10}$ Jean Yoyotte, „Pétoubastis III“, in: RdE 24, 1972, 216-223; zur Zählung der Könige namens Pedubaste vgl. Jürgen von Beckerath, „Beiträge zur Geschichte der Libyerzeit: 3. Die Könige namens Pedubaste", in: GM 147, 1995, 9-13 und ders., Handbuch der ägyptischen Königsnamen (MÄS 49; Mainz ${ }^{2} 1999$ ) $222-223$.

Vgl. Friedrich Karl Kienitz, Die politische Geschichte Ägyptens vom 7. bis zum 4. Jahrhundert vor der Zeitwende (Berlin 1953) 67-72.

${ }^{2} \mathrm{Zu}$ Chababasch vgl. u. a. Anthony Spalinger, "The Reign of King Chabbash: An Interpretation", in: ZÄS 105, 1978, 142-154; ders., „Addenda to "The Reign of King Chabbash: An Interpretation“ (ZÄS 105, 1978 , pp. 142-154)“, in: ZÄS 107, 1980, 87; Robert K. Ritner, „Khababash and the Satrap Stela - A Grammatical Rejoinder“", in: ZÄS 107, 1980, 135-137; Hans Goedicke, "Comments on the Satrap Stela", in: BES 6, 1985, 33-54; Thomas Schneider, Lexikon der Pharaonen (München 1996) 144-145.

${ }^{13}$ Vgl. Manfred G. Schmidt, Wörterbuch zur Politik (Stuttgart 1995) 982, wonach Usurpation als „widerrechtliche Inbesitznahme von Leitungspositionen in der Politik, z. B. durch Putsch, Staatsstreich oder Thronraub" definiert wird.

${ }^{14}$ Vgl. Herman De Meulenaere, Herodotos over de 26ste Dynastie (II,147-III,15) (Leuven 1951) 78-82; Elmar Edel, „Amasis und Nebukadrezar II.“, in: GM 29, 1978, 13-20; Alan B. Lloyd, Herodotus. Book II: Commentary 99-182 (Leiden 1988) 178-179; Anthony Leahy, ,The Earliest Dated Monument of Amasis and the End of the Reign of Apries", in: JEA 74, 1988, 183-199.

${ }^{15}$ Zur Lesung des Namens s. Willy Clarysse, „Nephorites, Founder of the 29th Dynasty and his Name“, in: CdE 69, 1994, 215-217.

${ }^{16}$ Vgl. E. G. Kraeling, The Brooklyn Museum Aramaic Papyri (New Haven 1953) no. 13; Claude Traunecker, „Essai sur l'histoire de la XXIX dynastie“, in: BIFAO 79, 1979, 398.
- Nektanebis (I.), der Nephorites II., den legitimen Nachfolger des Achoris, absetzte ${ }^{18}$,

- Nektanebos (II.), dessen Vater Tjahapimu ihn während eines Feldzugs seines Onkels Teos zum König erhob, und dem sich die Armee anschlo $\beta^{19}$.

Befreier von Fremdherrschaft: Das sind Psametik I. ${ }^{20}$ und Amyrtaios ${ }^{21}$, die jeweils als in Ägypten ansässige Potentaten den zuvor von Fremdherrschern besetzten Thron übernehmen konnten.

"Regelkonforme“ Thronfolger: Das sind solche Herrscher, die sich insbesondere durch patrilineare Erbfolge oder Designation legitimierten, ohne daß eine Gewaltanwendung gegen den vorherigen König erkennbar ist.

Obwohl diese Einteilung an manchen Positionen mit Unsicherheiten behaftet ist, soll nun der Versuch gewagt werden, verschiedene Auffälligkeiten in den Königsnamen zu untersuchen.

\section{Rückgriff auf alte Königsnamen}

Als erstes Fallbeispiel sei der Rückgriff auf Königsnamen vorgestellt, der wohl programmatisch und legitimierend interpretiert werden darf. Dabei setze ich voraus, daß Zugang zu den $\mathrm{Na}$ -

${ }^{17}$ John D. Ray, „Psammuthis and Hakoris", in: JEA 72, 1986, 149-158.

${ }^{18} \mathrm{Vgl}$. die Stele von Hermopolis (Günther Roeder, „Zwei hieroglyphische Inschriften aus Hermopolis“, in: ASAE 52, 391-392, C 9-11).

Vgl. Friedrich Karl Kienitz, Die politische Geschichte Ägyptens vom 7. bis zum 4. Jahrhundert vor der Zeitwende (Berlin 1953) 96-97 und Hanna Jenni, Elephantine XVII: Die Dekoration des Chnumtempels auf Elephantine durch Nektanebos II. (AV 90; Mainz 1998) 99-100.

${ }^{20}$ Vgl. die Überblicke bei Thomas Schneider, Lexikon der Pharaonen (München 1996) 310-312 und Peter Högemann, Das alte Vorderasien und die Achämeniden: ein Beitrag zur Herodot-Analyse (BTAVO 98; Wiesbaden 1992) 194-197.

${ }^{21}$ Vgl. Thomas Schneider, Lexikon der Pharaonen (München 1996) 106; A. Lemaire, „La fin de la première période perse en Égypte et la chronologie judéenne vers 400 av. J.-C.", in: Transeuphratène 9, Paris $1995,51-56$. 
men der früheren Könige herrschte - deren Namen und Titel beispielsweise in Bibliotheken nachlesbar waren ${ }^{22}$. Bei der Untersuchung dieses ersten Fallbeispiels werden Übereinstimmungen der ersten vier Namen der Spätzeitkönige mit ihren Amtsvorgängern berücksichtigt. Die Geburtsnamen werden außer acht gelassen, da ihnen, sofern sie tatsächlich noch die bei der Geburt vergebenen waren, noch keine Legitimationsbestrebungen durch Rückgriff zugekommen sein konnten ${ }^{23}$.

Genaue Übereinstimmungen von Titeln und Namen spätzeitlicher Könige mit denen früherer Könige liegen beispielsweise vor mit Nephorites I., dessen Horus-, Gold- und Thronnamen an Vorgänger aus der 26. bzw. 19. Dyn. anknüpften: Sein Horusname $3-i b$ entspricht dem Horusnamen Psametiks I., sein Goldname stp$n c ̌ r . w$ stimmt mit dem des Amasis überein, und sein Thronname $b 3-n i-R^{\complement} w$ mri-nčr.w geht auf denjenigen des Merenptah zurück.

Ein anderes anschauliches Beispiel ist der Horusname $s m 3-t 3$.wi des Kambyses, der dem des legendären Reichseinigers Mentuhotep II. entspricht - eine Anknüpfung an den ebenfalls diesen Namen führenden Sobekhotep I. scheint weniger wahrscheinlich zu sein.

Eine Aufzählung, welche „Herrscherkategorien" wieviele derartige Rückgriffe übten, zeigt eindeutige Tendenzen (Abb. 2): 35\% der Namen von Fremdherrschern, 33\% derer von Lokal- oder Gegenkönigen und immerhin noch $19 \%$ der Namen von Usurpatoren, aber nur $10 \%$ der Namen von ,regelkonformen“ Thronfolgern entsprechen denjenigen von Amtsvorgängern (die gesamte Belegübersicht ist in Anhang I wiedergegeben, die Auszählung nach "Herrscherkategorien" in Anhang II).

${ }^{22} \mathrm{Zu}$ Bibliotheken vgl. Günter Burkard, „Bibliotheken im Alten Ägypten", in: Bibliothek: Forschung und Praxis 4, München 1980, 79-115 und Jochem Kahl, Siut - Theben: zur Wertschätzung von Traditionen im alten Ägypten (PÄ 13; Leiden/ Boston/Köln 1999) 291-293.

${ }^{23}$ Eine mögliche Ausnahme ist Psametik III., der durch seinen Vater, den Usurpator Amasis, mit seinem Geburtsnamen, der dem des Dynastiegründers entspricht, in die legitime Thronfolge eingereiht wurde.

\begin{tabular}{|c|c|}
\hline „Herrscherkategorien“ & $\begin{array}{l}\text { Anzahl der Rückgriffe } \\
\text { auf einzelne Namen }\end{array}$ \\
\hline Fremdherrscher & $\begin{array}{c}35 \% \\
(19 / 54)\end{array}$ \\
\hline $\begin{array}{l}\text { Gegenkönige/ } \\
\text { Lokalkönige }\end{array}$ & $\begin{array}{c}33 \% \\
(4 / 12)\end{array}$ \\
\hline Usurpatoren & $\begin{array}{c}19 \% \\
(4 / 21)\end{array}$ \\
\hline $\begin{array}{l}\text { Befreier von } \\
\text { Fremdherrschaft }\end{array}$ & $\begin{array}{l}25 \% \\
(1 / 4)\end{array}$ \\
\hline $\begin{array}{l}\text { "regelkonforme“" } \\
\text { Thronfolger }\end{array}$ & $\begin{array}{c}10 \% \\
(3 / 28)\end{array}$ \\
\hline
\end{tabular}

Abb. 2. Häufigkeit der genauen Entsprechung von spätzeitlichen (25. Dyn. - Argeaden) Königsnamen mit denen von Vorgängern (vgl. im einzelnen Anhang I und II)

\section{Namentliche Bezugnahme auf Götter}

Das zweite Fallbeispiel ist die Erwähnung der Gottessohnschaft oder des Erwähltseins durch einen Gott in den Königsnamen.

Seit dem Alten Reich sicher nachweisbar wurde die Vorstellung vom König als Sohn des Re oder auch anderer Götter zum Zwecke seiner Legitimation benutzt ${ }^{24}$. Aus der Spätzeit seien als Beispiele genannt (alle Belege sind im Anhang III aufgelistet):

- Gemnefchonsbak, der sich in seinem Thronnamen als einen, „... den Re erzeugt hat" bezeichnete $^{25}$,

- Amasis, der sich als „.... Sohn der Neit", aber auch als „... Sohn des Osiris“" $\operatorname{sah}^{26}$, und

- Kambyses, der sich „Abkömmling des Re“ nannte ${ }^{27}$.

${ }^{24}$ Eberhard Otto, „Legitimation des Herrschens im pharaonischen Ägypten“", in: Saeculum 20, 1969, 404-407.

${ }^{25} s p s-k 3-R^{c} w$ iri.n- $n-R^{c} w(\mathrm{~T})$. Hier und im folgenden bezeichnen mit Jürgen von Beckerath, Handbuch der ägyptischen Königsnamen (MÄS 49; Mainz ${ }^{2}$ 1999) die Siglen $\mathrm{H}$ den Horusnamen, $\mathrm{N}$ den Nebtinamen, $\mathrm{G}$ den Goldnamen, $\mathrm{T}$ den Thronnamen und $\mathrm{E}$ den Geburts- bzw. Eigennamen.

${ }^{26}$ s3-Ni.t Spț-t3.wi (N), TCh-msin s3-Ni.t (E), TTh-msin s3Wsir (E).

${ }^{27} m s . t i w-R^{\top} w(\mathrm{~T})$. 


\begin{tabular}{|c|c|c|c|}
\hline "Herrscherkategorien“ & $\begin{array}{c}\text { Herrscher als } \\
\text { Abkömmling } \\
\text { der Götter }\end{array}$ & $\begin{array}{c}\text { Herrscher als } \\
\text { Auserwählter } \\
\text { der Götter }\end{array}$ & $\begin{array}{c}\text { Gesamt (Herrscher } \\
\text { als Abkömmling oder } \\
\text { Auserwählter der Götter) }\end{array}$ \\
\hline Fremdherrscher & $23 \%$ & $23 \%$ & $46 \%$ \\
Gegen-/Lokalkönige & $(3 / 13)$ & $(3 / 13)$ & $(6 / 13)$ \\
\hline Usurpatoren & $22 \%$ & $11 \%$ & $33 \%$ \\
& $(2 / 9)$ & $(1 / 9)$ & $80 \%$ \\
\hline Befreier von Fremdherrschaft & $40 \%$ & $80 \%$ & $(4 / 5)$ \\
\hline "regelkonforme“ Thronfolger & $(2 / 5)$ & $(4 / 5)$ & $0 \%$ \\
& $(0 / 2)$ & $0 \%$ & $(0 / 2)$ \\
\hline
\end{tabular}

Abb. 3. Häufigkeit der Nennung der Gottessohnschaft bzw. der Auserwählung durch die Götter in spätzeitlichen Königsnamen

In engem Bezug zu dem Prinzip der Gottessohnschaft steht das der Auserwähltheit des Königs, ägyptisch durch stp bezeichnet ${ }^{28}$. Zwar wurde oft von Königen behauptet, daß sie Auserwählte eines Gottes seien, aber ein besonderes Augenmerk verdienen die Fälle, in denen dies ausdrücklich im Namen festgehalten wurde. Auch hier einige Beispiele (vgl. Anhang IV mit der kompletten Übersicht):

- Amasis bezeichnete sich als „Auserwählter der Götter ${ }^{c^{29}}$,

- Chababasch als ,auserwähltes Abbild des Ptah $^{\text {“ }}{ }^{30}$ und

- Alexander d. Gr. als „Geliebter des Re, den Amun auserwählt hat ${ }^{63^{31}}$.

$\mathrm{Da}$ die Königsnamen sich insgesamt auf verschiedene Aufgaben- und Wesensbereiche des Herrschers bezogen (z. B. auf das Durchsetzen der Maat, die Sicherung der Grenzen, die Pflege der Götterkulte oder die göttliche Abstammung), wird im folgenden die Anzahl der Herr-

${ }^{28}$ Die Belege für Namen mit dem Bestandteil mri "Geliebter" sind hier nicht berücksichtigt, da mri nur in Verbindung mit der Präposition $r$ als ,erwählen “ übersetzt werden sollte (zu mri $r$ vgl. Siegfried Morenz, „Die Erwählung zwischen Gott und König in Ägypten", in: Sino-Japonica. Festschrift André Wedemeyer zum 80. Geburtstag (Leipzig 1956) 118-125).

${ }^{29}$ stp-nčr.w (G).

${ }^{30}$ snn-stp-ni-Pth $(\mathrm{T})$.

${ }^{31}$ mri-R $R^{\top}$ stp.n-Imn.w (T); vgl. zur Problematik der Lesung Herman De Meulenaere, „Le protocole royal de Philippe Arrhidée“, in: CRIPEL 13, 1991, 57. scher ausgezählt, die in ihren Namen den Aspekt der göttlichen Auserwähltheit oder Sohnschaft betonten (unabhängig davon, ob dies ein- oder mehrmals geschah). Es ergibt sich dann das in Abb. 3 dargestellte Bild ${ }^{32}$, bei dem besonders auffällt, daß $80 \%$ (4 von 5 ) der Usurpatoren, aber nur $42 \%$ (3 von 7) der ,regelkonformen " Thronfolger sich als Abkömmlinge oder Auserwählte der Götter bezeichneten.

Es zeigt sich mit dem außerordentlich hohen prozentualen Anteil der Usurpatoren deren besondere Neigung, ihre Beziehung zu den Göttern zu betonen und dadurch ihr Legitimationsdefizit bezüglich ihrer Abstammung auszugleichen.

\section{Namen mit Erwähnung des Schutzes des Landes}

Als letztes Beispiel seien die Königsnamen vorgestellt, die sich ausdrücklich auf das Schlagen der Feinde oder den Schutz Ägyptens beziehen $^{33}$.

${ }^{32}$ Die Anzahl der Herrscher umfaßt alle namentlich belegten, also auch die, welche nur mit Eigennamen belegt sind, da auch an die Eigennamen nachträglich Epitheta entsprechenden Inhalts angefügt und in Kartusche geschrieben werden konnten.

${ }^{33}$ Bei der prozentualen Auszählung sind nur Herrscher berücksichtigt, die mindestens einen der ersten vier Thronnamen aufweisen. 


\begin{tabular}{|c|c|c|c|}
\hline "Herrscherkategorien“ & Schutz nach innen & $\begin{array}{c}\text { Schutz/Angriff } \\
\text { nach außen }\end{array}$ & Schutz insgesamt \\
\hline Fremdherrscher & $20 \%$ & $30 \%$ & $40 \%$ \\
$(2 / 10)$ & $(3 / 10)$ & $(4 / 10)$ \\
\hline Gegen-/Lokalkönige & $0 \%$ & $0 \%$ & $0 \%$ \\
& $(0 / 8)$ & $(0 / 8)$ & $(0 / 8)$ \\
\hline Usurpatoren & $20 \%$ & $20 \%$ & $20 \%$ \\
& $(1 / 5)$ & $(1 / 5)$ & $0 \%$ \\
\hline Befreier von Fremdherrschaft & $0 \%$ & $0 \%$ & $(0 / 1)$ \\
\hline "regelkonforme" Thronfolger & $(0 / 1)$ & $(0 / 1)$ & $16 \%$ \\
& $16 \%$ & $16 \%$ & $(1 / 6)$ \\
\hline
\end{tabular}

Abb. 4. Prozentualer Anteil von Königen, die explizit „Schutz“ oder „Aggression gegen außen“ in ihren Namen erwähnen, unterteilt nach „Herrscherkategorien“

Zunächst Beispiele für Namen, die den Schutz Ägyptens hervorheben (vgl. Anhang V) ${ }^{34}$ :

- Taharqa war „einer, der die beiden Länder beschützt ${ }^{\text {c } 35}$,

- Nektanebos (II.) „einer, der die beiden Länder liebt und Ägypten beschützt ${ }^{\text {* }^{36}}$,

- Alexander der Große „einer, der Ägypten beschützt ${ }^{6 c^{37}}$.

Nun Beispiele für Namen, die eine Aggression gegen Feinde explizit aufgreifen (vgl. Anhang VI):

- Schebitko wurde in seinem Goldnamen als „einer mit großer Schlagkraft, der die Neun Bogen schlägt" bezeichnet ${ }^{38}$,

- Teos als „einer, der Ägypten schützt und die Fremdländer niederzwingt ${ }^{6{ }^{c 3}}$.

- Alexanders des Großen Horusname lautete „tapferer Herrscher, der den Fremdländern nahekommt ${ }^{\text {c } 40}$.

Nach Herrscherkategorien geordnet zeigt sich (Abb. 4), daß die Fremdherrscher den

${ }^{34}$ Vgl. auch die unsicheren Fälle: Pije: H s:htp-

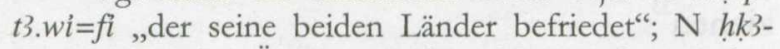
$K m . t$ „Herrscher Ägyptens“ (diese Namen deuten eher auf eindeutigen Machtanspruch über Ägypten); Gemnefchonsbak: $\mathrm{H} s:{ }^{\complement}$ nh-t $3 . w i$, der die beiden Länder leben läßt" (dieser Name kann auf die Versorgung des Landes bezogen werden).

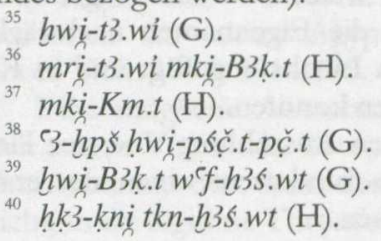

Schutz des Landes wesentlich deutlicher in den Vordergrund stellten als indigene Thronfolger seien es Usurpatoren oder „regelkonforme“: 4 von 10 Fremdherrschern, insgesamt $40 \%$ also, nahmen in einem ihrer ersten vier Namen auf diese Thematik bezug, hingegen nur 1 von 6 „regelkonformen“ Thronfolgern, also 16,6\%. Gegen- und Lokalkönige gaben in ihren Namen nicht vor, für das Land schützend tätig sein zu können, was ein gesundes $\mathrm{Ma} \beta$ an Realität erkennen läßt.

\section{Resumée}

Die soeben vorgestellten Beispiele mögen genügen, um zu erkennen, daß Usurpatoren und Fremdherrscher offensichtlich einem stärkeren Legitimationsdruck ausgesetzt waren als ,regelkonforme" Thronfolger und daher verstärkt an die Amtsvorgänger (durch Namensgleichheit) bzw. an die Götter (durch das Prinzip der Gottessohnschaft bzw. der Auserwähltheit) anzuknüpfen versuchten oder den sicherheitspolitischen Bereich (innen wie außen) besonders betonten. Diese Ergebnisse lassen auch in anderen Bereichen des spätzeitlichen Königtums große Forschungschancen in der Thematik "Thronwechsel und Usurpationen" vermuten.

\section{ANHANG I}

Königsnamen der Spätzeit, die mit denen der Vorgänger übereinstimmen, unter Angabe der Gesamtzahl der Königsnamen pro Herrscher und 
Dynastie aufgeteilt in die ersten vier Namen (Horusname, Nebtiname, Goldname, Thronname) und darauf folgend in runden Klammern die Anzahl der Geburts- bzw. Eigennamen sowie unter Angabe der Anzahl der genauen Entsprechungen zu älteren Namen und Titeln ${ }^{41}$ :

\begin{tabular}{|c|c|c|}
\hline 25. Dynastie & $\begin{array}{l}\text { Anzahl der } \\
\text { ersten vier } \\
\text { Namen } \\
\text { (+ Geburts- } \\
\text { namen) }\end{array}$ & $\begin{array}{c}\text { Anzahl der } \\
\text { genauen } \\
\text { Entsprechungen }\end{array}$ \\
\hline $\begin{array}{c}\text { Kaschta } \\
\text { (vor ca. 746) }\end{array}$ & $1(+1)$ & 1 \\
\hline $\begin{array}{c}\text { Pije (ca. } \\
746-715 / 713)\end{array}$ & $6(+4)$ & 2 \\
\hline $\begin{array}{c}\text { Schabako } \\
(715 / 713- \\
700 / 698)\end{array}$ & $5(+2)$ & 1 \\
\hline $\begin{array}{c}\text { Schebitko } \\
(700 / 698-690)\end{array}$ & $11(+3)$ & 8 \\
\hline $\begin{array}{c}\text { Taharqa } \\
(690-664)\end{array}$ & $7(+2)$ & 2 \\
\hline $\begin{array}{c}\text { Tanotamun } \\
(664-\text { ca. } 655)\end{array}$ & $2(+1)$ & - \\
\hline Insgesamt & $32(+13)$ & 14 \\
\hline
\end{tabular}

Belege (25. Dyn.):

- Kaschta:

T $m 3^{\complement}-R^{\top} w$ (= T Sebekhotep IX., 13. Dyn.) oder T $n . i-m 3^{\top} . t-R^{\top} w$ (= T Amenemhet III., 12. Dyn.) ${ }^{42}$.

- Pije:

H k3-nht hri-m-W3s.t (= H Thutmosis III., 18. Dyn.; H Ramses IX., 20. Dyn.; H Pinudjem I., 21. Dyn.; H Osorkon II., 22. Dyn.; H Takelot II., 22. Dyn.; H Osorkon III., 22. Dyn.; H Harsiese, 22. Dyn.; vgl. H Schebitko 25. Dyn.);

T $w s r-M 3^{\complement} . t-R^{\top} w$ (= T Ramses II., 19. Dyn.; T Amenemope, 21. Dyn.; T Osorkon III., 22. Dyn.; T Takelot III., 22. Dyn.; T Rudamun, 22. Dyn.; vgl. weitere Namen dieser Art mit Epitheta, alle nicht vor Ramses II.).

- Schabako:

T $n f r-k 3-R^{e} w$ (= T Pepi II., 6. Dyn.; T Neferkare, 8. Dyn.; T Neferkare Nebi, 8. Dyn.; T Neferkare Chendu, 8. Dyn.; T Neferkare Tereru, 8. Dyn.; T Neferkare Pepisonbe, 8. Dyn.; T Neferkare,

${ }^{41}$ Grundlage für die Vergleiche der Namen war Jürgen von Beckerath, Handbuch der ägyptischen Königsnamen (MÄS 49; Mainz ${ }^{2}$ 1999). Folgende Siglen gelten: $\mathrm{H}=$ Horusname, $\mathrm{N}=$ Nebtiname, $\mathrm{G}=$ Goldname, $\mathrm{T}=$ Thronname, $\mathrm{E}=$ Geburts- bzw. Eigenname.

${ }^{42}$ Zur Lesung vgl. von Beckerath, Handbuch der Königsnamen, 206 (mit Anm. 2).
9./10. Dyn.; T Pefthew awbaste von Herakleopolis, 24. Dyn., Lokalkönig).

- Schebitko:

H $c ̌ t-h h^{c} w(=$ H Djedkare, 5. Dyn.);

H k3-nht h hi-m-W3s.t (= H Thutmosis III., 18.

Dyn.; H Ramses IX., 20. Dyn.; H Pinudjem I., 21.

Dyn.; H Osorkon II., 22. Dyn.; H Takelot II., 22.

Dyn.; H Osokon III., 22. Dyn.; H Harsiese, 22.

Dyn.; H Pije, 25. Dyn.);

N 3-šfi.t-m-t3.w-nb.w (= N Thutmosis III., 18.

Dyn.);

N s:hi-M $i 3^{\top} . t$ mri-t3.wi (= N Thutmosis III., 18. Dyn.);

$\mathrm{N} c \grave{c t}-h^{c} w-N b . t i$ (= N Djedkare, 5. Dyn.);

G 3-hpš hwwi-pśč.t-pč.t (= G Thutmosis III., 18.

Dyn.);

G hrw-hr-nht.w (= G Thutmosis III., 18. Dyn.); T čt $-k 3-R^{\top} w$ (= T Djedkare, 5. Dyn.; T Djedkare Schemai, 8. Dyn.; T ${ }^{\mathrm{c}}$ Anati, 14. Dyn.).

- Taharqo:

G bik-nbw (= G Djoser, 3. Dyn. (postum); G Snofru, 4. Dyn.; G Teti, 6. Dyn.; G Meriibre Achtoi (?), 9./10. Dyn.; G Mentuhotep II., 11. Dyn.; G Tefnacht, 24. Dyn.);

G bik.wi-nbw (= G Cheops, 4. Dyn.; G Merenre, 6. Dyn.).

\begin{tabular}{|c|c|c|}
\hline $\begin{array}{l}\text { Lokalkönige } \\
\text { im Delta } \\
\text { (parallel zur } \\
\text { 25. Dyn.) }\end{array}$ & $\begin{array}{c}\text { Anzahl der } \\
\text { ersten vier } \\
\text { Namen } \\
\text { (+ Geburts- } \\
\text { namen) }\end{array}$ & $\begin{array}{c}\text { Anzahl der } \\
\text { genauen } \\
\text { Entspre- } \\
\text { chungen }\end{array}$ \\
\hline Pedubaste III. & $\begin{array}{c}3(+1) \\
{[+1 \text { fragment. } H]}\end{array}$ & 1 \\
\hline Gemnefchonsbak & $2(+2)$ & - \\
\hline Neferkare I $[\ldots]$ & $\begin{array}{c}2(+1) \\
{[+1 \text { fragment. } T]}\end{array}$ & 1 \\
\hline Penamun & $1(+1)$ & - \\
\hline $\begin{array}{c}\text { Nechepsos von } \\
\text { Sais }\end{array}$ & $1(+1)$ & $1(?)$ \\
\hline Necho I. von Sais & $1(+1)$ & 1 \\
\hline Insgesamt & $\begin{array}{c}10(+7) \\
{[+2 \text { fragmentierte }} \\
\text { Namen }]\end{array}$ & 4 \\
\hline
\end{tabular}

Belege (Lokalkönige im Delta) ${ }^{43}$ :

- Pedubaste III.:

T s:htp-ib-R ${ }^{r} w$ (= T Amenemhet I., 12. Dyn.;

T Sehetepibre, 13. Dyn.; T Harnedjherjotef (?), 13. Dyn.).

${ }^{43}$ Zum Thronnamen sps $-k 3-R^{\complement} w$ iri.n- $R^{\varsigma} w$ des Gemnefchonsbak vgl. T $s p s s-k 3-R^{r} w$ des Königs Schepseskare aus der 5. Dyn. (von Beckerath, Handbuch der Königsnamen, 56, Anm. 4). 
- Neferkare:

T $n f r-k 3-R^{\top} w$ (= T Pepi II., 6. Dyn.; T Neferkare, 8. Dyn.; T Neferkare, 9./10. Dyn.; T Pefthew'awbaste von Herakleopolis, 24. Dyn. Lokalkönig; T Schabako, 25. Dyn.).

- Nechepsos von Sais:

$\mathrm{T}$ (?) $m n-i b-R^{c} w$ (= T Menibre, 14. Dyn.).

- Necho I. von Sais:

T $m n-h p r-R^{\top} w$ (= T Thutmosis III., 18. Dyn.).

\begin{tabular}{|c|c|c|}
\hline 26. Dynastie & $\begin{array}{c}\text { Anzahl der } \\
\text { ersten vier } \\
\text { Namen } \\
\text { (+ Geburts- } \\
\text { namen) }\end{array}$ & $\begin{array}{l}\text { Anzahl der } \\
\text { genauen } \\
\text { Entsprechungen }\end{array}$ \\
\hline $\begin{array}{l}\text { Psametik I. } \\
(664-610)\end{array}$ & $4(+2)$ & 1 \\
\hline $\begin{array}{l}\text { Necho II. } \\
(610-595)\end{array}$ & $5(+2)$ & - \\
\hline $\begin{array}{l}\text { Psametik II. } \\
(595-589)\end{array}$ & $6(+2)$ & 1 \\
\hline $\begin{array}{c}\text { Apries } \\
(589-570)\end{array}$ & $5(+1)$ & - \\
\hline $\begin{array}{c}\text { Amasis } \\
(570-526)\end{array}$ & $4(+3)$ & - \\
\hline $\begin{array}{l}\text { Psametik III. } \\
(526-525)\end{array}$ & $2(+1)$ & 1 \\
\hline Insgesamt & $26(+11)$ & 3 \\
\hline
\end{tabular}

Belege (26. Dyn.):

- Psametik I.:

$\mathrm{T} w 3 h-i b-R^{c} w$ (= T Ja ib, 13. Dyn.).

- Psametik II.:

T $n f r-i b-R^{\top} w$ (= T Neferibre, 14. Dyn.).

- Psametik III. ${ }^{44}$ :

$\mathrm{T}^{\top} n h-k 3-R^{\top} w$ (= T Anchkare, 14. Dyn.).

\begin{tabular}{|c|c|c|}
\hline 27. Dynastie & $\begin{array}{c}\text { Anzahl der } \\
\text { ersten vier } \\
\text { Namen } \\
\text { (+ Geburts- } \\
\text { namen) }\end{array}$ & $\begin{array}{c}\text { Anzahl der } \\
\text { genauen } \\
\text { Entsprechungen }\end{array}$ \\
\hline $\begin{array}{c}\text { Kambyses } \\
(525-522)\end{array}$ & $2(+1)$ & 1 \\
$\begin{array}{c}\text { Dareios I. } \\
(522 / 21-486 / 85) \\
\text { Xerxes I. }\end{array}$ & $5(+1)$ & 1 \\
$(486 / 85-465 / 64)$ & $-(+1)$ & - \\
\hline
\end{tabular}

${ }^{44}$ Vgl. auch den Eigennamen pśmčk, der dem des Dynastiegründers entspricht; der Sohn des Usurpators Amasis wird dadurch in die legitime Thronfolge eingereiht.

\begin{tabular}{c|c|c|} 
Artaxerxes I. & $-(+1)$ & - \\
$(465 / 64-424)$ & & - \\
Xerxes II. & - & \\
$(424 / 23)$ & & - \\
Dareios II. & - & - \\
$(423-405 / 04)$ & & - \\
Artaxerxes II. & - & 2 \\
$(405 / 04-401)$ & & \\
\hline Insgesamt & $7(+4)$ & \\
\hline
\end{tabular}

Belege (27. Dyn.):

- Kambyses:

H sm3-t3.wi ( $=\mathrm{H}$ Mentuhotep II., 11. Dyn.; H Sobekhotep I., 13. Dyn.).

- Dareios I.:

H $m n h-i b$ (= H Psametik II., 26. Dyn.).

\begin{tabular}{|c|c|c|}
\hline $\begin{array}{c}\text { Ägyptische } \\
\text { Gegenkönige } \\
\text { zur 27. Dynastie }\end{array}$ & $\begin{array}{c}\text { Anzahl der } \\
\text { ersten vier } \\
\text { Namen } \\
\text { (+ Geburts- } \\
\text { namen) }\end{array}$ & $\begin{array}{c}\text { Anzahl der } \\
\text { genauen } \\
\text { Entsprechungen }\end{array}$ \\
\hline Pedubaste IV. & $1(+1)$ & - \\
Inaros & $-(+1)$ & - \\
Insgesamt & $1(+2)$ & - \\
\hline
\end{tabular}

\begin{tabular}{|c|c|c|}
\hline 28. Dynastie & $\begin{array}{c}\text { Anzahl der } \\
\text { ersten vier } \\
\text { Namen } \\
(+ \text { Geburts- } \\
\text { namen })\end{array}$ & $\begin{array}{c}\text { Anzahl der } \\
\text { genauen } \\
\text { Entsprechungen }\end{array}$ \\
\hline $\begin{array}{c}\text { Amyrtaios } \\
(404 / 01-399)\end{array}$ & $-(+1)$ & - \\
\hline
\end{tabular}

\begin{tabular}{|c|c|c|}
\hline 29. Dynastie & $\begin{array}{c}\text { Anzahl der } \\
\text { ersten vier } \\
\text { Namen } \\
\text { (+ Geburts- } \\
\text { namen) }\end{array}$ & $\begin{array}{c}\text { Anzahl der } \\
\text { genauen } \\
\text { Entsprechungen }\end{array}$ \\
\hline $\begin{array}{c}\text { Nephorites I. } \\
(399-393)\end{array}$ & $3(+1)$ & 3 \\
\hline $\begin{array}{c}\text { Achoris } \\
(393-380)\end{array}$ & $6(+1)$ & 1 \\
\hline $\begin{array}{l}\text { Psamuthis } \\
(393 / 92)\end{array}$ & $2(+1)$ & - \\
\hline $\begin{array}{c}\text { Nephorites II. } \\
(380)\end{array}$ & $-(+1)^{45}$ & - \\
\hline Insgesamt & $11(+4)$ & 4 \\
\hline
\end{tabular}

${ }^{45}$ Der einzige Beleg in der Demotischen Chronik ist nicht bei von Beckerath, Handbuch der Königsnamen erwähnt. 
Belege (29. Dyn.):

- Nephorites I.:

H 3-ib (= H Psametik I., 26. Dyn.);

G stp-nčr.w (= G Amasis, 26. Dyn.);

$\mathrm{T} b 3-n i-R^{\ulcorner} w$ mrin-nčr.w (= T Merenptah,

19. Dyn.).

- Achoris :

G s:htp-nčr.w (= G Petubastis III., Lokalkönig 25. Dyn.).

\begin{tabular}{|c|c|c|}
\hline 30. Dynastie & $\begin{array}{c}\text { Anzahl der } \\
\text { ersten vier } \\
\text { Namen } \\
(+ \text { Geburts- } \\
\text { namen) }\end{array}$ & $\begin{array}{c}\text { Anzahl der } \\
\text { genauen } \\
\text { Entsprechungen }\end{array}$ \\
\hline $\begin{array}{c}\text { Nektanebis (I.) } \\
(380-362) \\
\text { Teos } \\
(364 / 62-360) \\
\text { Nektanebos (II.) } \\
(360-342)\end{array}$ & $4(+1)$ & - \\
\hline Insgesamt & $16(+1)$ & - \\
\hline
\end{tabular}

Belege (30. Dyn.):

- Nektanebis (I.):

T hpr-k3-R ${ }^{\top} w$ (= T Sesostris I., 12. Dyn.).

\begin{tabular}{|c|c|c|}
\hline 31. Dynastie & $\begin{array}{c}\text { Anzahl der } \\
\text { ersten vier } \\
\text { Namen } \\
\text { (+ Geburts- } \\
\text { namen) }\end{array}$ & $\begin{array}{c}\text { Anzahl der } \\
\text { genauen } \\
\text { Entsprechungen }\end{array}$ \\
\hline $\begin{array}{c}\text { Artaxerxes III. } \\
\text { Ochos (342-338) } \\
\text { Arses (Aroges) } \\
(338-336) \\
\text { Dareios III. } \\
(336-332 / 30)\end{array}$ & - & - \\
\hline Insgesamt & $-(+1)$ & - \\
\hline
\end{tabular}

\begin{tabular}{|c|c|c|}
\hline $\begin{array}{c}\text { Ägyptischer } \\
\text { Gegenkönig zur } \\
\text { 31. Dynastie }\end{array}$ & $\begin{array}{c}\text { Anzahl der } \\
\text { ersten vier } \\
\text { Namen } \\
\text { (+ Geburts- } \\
\text { namen })\end{array}$ & $\begin{array}{c}\text { Anzahl der } \\
\text { genauen } \\
\text { Entsprechungen }\end{array}$ \\
\hline $\begin{array}{c}\text { Chababasch } \\
(338 / 37-336 / 35)\end{array}$ & $1(+1)$ & - \\
\hline
\end{tabular}

${ }^{46}$ Zum Horusnamen 3-ib mri-t3.wi vgl. 3-ib $\mathrm{H}$ Psametik I., 26. Dyn. und H Nephorites I., 29. Dyn.; der Nebtiname knw entspricht dem Goldnamen Psametiks I., 26. Dyn.

\begin{tabular}{|c|c|c|}
\hline Argeaden & $\begin{array}{c}\text { Anzahl der } \\
\text { ersten vier } \\
\text { Namen } \\
\text { (+ Geburts- } \\
\text { namen })\end{array}$ & $\begin{array}{c}\text { Anzahl der } \\
\text { genauen } \\
\text { Entsprechungen }\end{array}$ \\
\hline $\begin{array}{c}\text { Alexander } \\
\text { der Große } \\
\text { Philippos } \\
\text { Arrhidaios } \\
\text { Alexander II. } \\
\text { (IV.) }\end{array}$ & $4(+1)$ & - \\
\hline Insgesamt & $6(+1)$ & - \\
\hline
\end{tabular}

Belege (Argeaden) ${ }^{47}$ :

- Philippos Arrhidaios ${ }^{48}$ :

$\mathrm{H} w 3 c_{c}-t 3 . w i^{49}$ (= H Unas, 5. Dyn., H Takeloth III., 22. Dyn. (oberäg.));

H k3-nht mri-M $3^{\top} . t$ (= H Thutmosis I., 18. Dyn.; Ramses II., 19. Dyn.; Tausret, 19. Dyn.; Siamun, 21. Dyn.; Osorkon II., 22. Dyn.; Scheschonq III., 22. Dyn.);

T mri-R ${ }^{\top} w$ stp.n-Imn.w (= T Alexander d. Gr.).

\section{ANHANG II}

Gesamtzahl der spätzeitlichen Königsnamen, geordnet nach Fremdherrschern, Gegen-/Lokalkönigen, Usurpatoren, Befreiern und „regelkonformen“ Thronfolgern sowie aufgeteilt in die ersten vier $\mathrm{Na}$ men und darauf folgend in runden Klammern die Anzahl der Eigennamen gefolgt von der Angabe der Anzahl der genauen Entsprechungen mit Namen und Titeln von Vorgängern.

\begin{tabular}{|c|c|c|}
\hline Fremdherrscher & $\begin{array}{c}\text { Anzahl der } \\
\text { ersten 4 Namen } \\
\text { (+ Geburts- } \\
\text { namen) }\end{array}$ & $\begin{array}{c}\text { Anzahl der } \\
\text { genauen } \\
\text { Entsprechungen }\end{array}$ \\
\hline 25. Dynastie & $32(+13)$ & 14 \\
27. Dynastie & $7(+4)$ & 2 \\
31. Dynastie & $-(+1)$ & - \\
Argeaden & $15(+3)$ & 3 \\
\hline $\begin{array}{c}\text { Fremdherrscher } \\
\text { Gesamt }\end{array}$ & $54(+21)$ & $19 \rightarrow 35 \%$ \\
\hline
\end{tabular}

${ }^{47}$ Zum Horusnamen Alexanders d. Gr. (mki-Km.t) vgl. den Horusnamen $k 3-n h t$ mki-Km.t Sethos II. Zum Thronnamen Alexanders des Großen $m r i-R^{\top} w$ stp.nImn.w (De Meulenaere, CRIPEL 13, 1991, 57) vgl. mri.y- $R^{\complement} w$ (Thronname Pepis I.). Zum Thronnamen Alexanders II. (IV.) $h^{\complement c}-i b-R^{\complement} w$ stp.n-Imn.w vgl. den Thronnamen $h^{e c}-i b-R^{c} w$ des Apries.

${ }^{48} \mathrm{Vgl}$, auch den Thronnamen mri-k3-R ${ }^{\complement} w$ stp.n-Imn.w mit demjenigen des Merikare (9./10. Dyn.; $\left.m r i-k 3-R^{\complement} w\right)$.

${ }^{49}$ Alfred Hermann, in: Kurt Bittel - Alfred Hermann, „Grabungsbericht Hermopolis 1933“, in: MDAIK 5, 1934, 42 (Anm. 1); vgl. De Meulenaere, CRIPEL 13, 1991, 53. 


\begin{tabular}{|c|c|c|}
\hline $\begin{array}{c}\text { Gegenkönige/ } \\
\text { Lokalkönige }\end{array}$ & $\begin{array}{c}\text { Anzahl der } \\
\text { ersten } 4 \text { Namen } \\
\text { (+ Geburts- } \\
\text { namen })\end{array}$ & $\begin{array}{l}\text { Anzahl der } \\
\text { genauen } \\
\text { Entsprechungen }\end{array}$ \\
\hline $\begin{array}{c}\text { Lokalkönige im } \\
\text { Delta }\end{array}$ & $\begin{array}{l}10(+7) \\
{[+2 \text { fragmen- }} \\
\text { tierte Namen] }\end{array}$ & 4 \\
\hline $\begin{array}{l}\text { ägyptische } \\
\text { Gegenkönige zur } \\
\text { 27. Dynastie }\end{array}$ & $1(+2)$ & - \\
\hline $\begin{array}{l}\text { ägyptischer } \\
\text { Gegenkönig zur } \\
\text { 31. Dynastie }\end{array}$ & $1(+1)$ & - \\
\hline $\begin{array}{c}\text { Gegenkönige } \\
\text { Gesamt }\end{array}$ & $12(+10)$ & $4 \rightarrow 33 \%$ \\
\hline
\end{tabular}

\begin{tabular}{|c|c|c|}
\hline $\begin{array}{c}\text { Befreier } \\
\text { von Fremd- } \\
\text { herrschaft }\end{array}$ & $\begin{array}{c}\text { Anzahl der } \\
\text { ersten 4 Namen } \\
\text { (+ Geburts- } \\
\text { namen) }\end{array}$ & $\begin{array}{c}\text { Anzahl der } \\
\text { genauen } \\
\text { Entsprechungen }\end{array}$ \\
\hline $\begin{array}{c}\text { Psametik I. } \\
\text { Amyrtaios }\end{array}$ & $\begin{array}{c}4(+2) \\
-(+1)\end{array}$ & 1 \\
\hline $\begin{array}{c}\text { Befreier von } \\
\text { Fremdherrschaft } \\
\text { Gesamt }\end{array}$ & $4(+3)$ & $1 \rightarrow 25 \%$ \\
\hline
\end{tabular}

\begin{tabular}{|c|c|c|}
\hline Usurpatoren & $\begin{array}{c}\text { Anzahl der } \\
\text { ersten 4 Namen } \\
\text { (+ Geburts- } \\
\text { namen) }\end{array}$ & $\begin{array}{c}\text { Anzahl der } \\
\text { genauen } \\
\text { Entsprechungen }\end{array}$ \\
\hline Amasis & $4(+3)$ & - \\
Nephorites I. & $3(+1)$ & 3 \\
Psamuthis & $2(+1)$ & - \\
Nektanebis (I.) & $4(+1)$ & 1 \\
Nektanebos (II.) & $8(+6)$ & - \\
\hline Usurpatoren & $21(+12)$ & 4 \\
Gesamt & & \\
\hline
\end{tabular}

\begin{tabular}{|c|c|c|}
\hline $\begin{array}{c}\text { „Regel- } \\
\text { konforme“ } \\
\text { Thronfolger }\end{array}$ & $\begin{array}{c}\text { Anzahl der } \\
\text { ersten 4 Namen } \\
\text { (+ Geburts- } \\
\text { namen) }\end{array}$ & $\begin{array}{c}\text { Anzahl der } \\
\text { genauen } \\
\text { Entsprechungen }\end{array}$ \\
\hline Necho II. & $5(+2)$ & - \\
Psametik II. & $6(+2)$ & 1 \\
Apries & $5(+1)$ & - \\
Psametik III. & $2(+1)$ & 1 \\
Achoris & $6(+1)$ & 1 \\
Nephorites II. & $-(+1)$ & - \\
Teos & $4(+1)$ & - \\
\hline "Regelkonforme" \\
Thronfolger \\
Gesamt
\end{tabular}

Alternativzählung

\begin{tabular}{|c|c|c|}
\hline "Regelkonforme“ & $32(+12)$ & $4 \rightarrow 12 \%$ \\
Thronfolger + & & \\
Befreier von \\
Fremdherrschaft \\
Gesamt
\end{tabular}

\section{ANHANG III}

Königsnamen, die den Herrscher als Sohn bzw. Nachkommen einer Gottheit nennen:

\begin{tabular}{|c|c|c|}
\hline Pije & E py mri-Imn.w s3-B3s.tt & „.... Sohn der Bastet ${ }^{\varsigma 6}$ \\
\hline Gemnefchonsbak & T spss-k3-R $R^{c} w$ iri. $n-R^{c} w$ & „... den Re erzeugt hat" \\
\hline Apries & G s3-Pth $m r i=f$ & „Sohn des Ptah ...“ \\
\hline Amasis & $\begin{array}{c}\text { N s3-Ni.tspt-t3.wi } \\
\text { E TTh-msi s3-Ni.t } \\
\text { E Trh-msị s3-Wsir }\end{array}$ & $\begin{array}{l}\text { „... Sohn der Neit" } \\
\text { „... Sohn der Neit }{ }^{“} \\
\text {,..Sohn des Osiris“" }\end{array}$ \\
\hline Kambyses & $\mathrm{T} m s . t i w-R^{\top} w$ & „Abkömmling des $\mathrm{Re}^{“}$ \\
\hline Dareios I. & $\mathrm{T}$ stw.t- $R^{r} w$ & „Sprößling des $\mathrm{Re}^{“}$ \\
\hline Pedubaste IV. & E $p 3-t i t h-B 3 s . t t s 3(-B 3 s . t t)$ & „... Sohn (der Bastet) ${ }^{c}$ \\
\hline Nektanebos (II.) & $\begin{array}{c}\text { E } n h t-H r . w-(n)-H b y . t m r i-1 n h r \\
\text { s3-Hw.t-Hr.w } \\
\text { E } n h t-H r . w-(n)-H b y . t \text { s3-mri-Hw.t-Hr.w} \\
\text { E } n h t-H r . w-(n)-H b y . t \text { mrin-Inhr s3-B3s.tt }\end{array}$ & $\begin{array}{l}\text { „... Sohn der Hathor" } \\
\text { „... geliebter Sohn der Hathor“ } \\
\text { „... Sohn der Bastet" }\end{array}$ \\
\hline
\end{tabular}


ANHANG IV

Königsnamen, die den Herrscher als Auserwählten einer Gottheit bzw. als auserwähltes Abbild eines Gottes bezeichnen:

\begin{tabular}{|c|c|c|}
\hline Amasis & G stp-nčr.w & „Auserwählter der Götter“ \\
\hline Nephorites I. & G stp-nčr.w & „Auserwählter der Götter“ \\
\hline Psamuthis & T wsr-R`w stp.n-Pth & „... der, den Ptah auserwählt hat"“ \\
\hline Achoris & 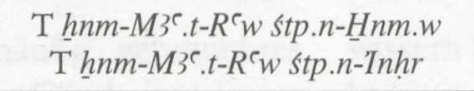 & $\begin{array}{l}\text { „... der, den Chnum auserwählt hat" } \\
\text { „.. der, den Onuris auserwählt hat" }\end{array}$ \\
\hline Teos & E čtt-Hr.w stp.n-Inhrr & „... der, den Onuris auserwählt hat “ \\
\hline Nektanebos (II.) & $\begin{array}{c}\text { T s:nçm-ib-R } R^{\top} w \text { stp.n-Inhr } \\
\text { T s:nçm-ib- } R^{\top} w \text { stp.n-Hw.t-Hr.w }\end{array}$ & $\begin{array}{l}\text { „... der, den Onuris auserwählt hat" } \\
\text { „... der, den Hathor auserwählt hat }\end{array}$ \\
\hline Chababasch & T snn-stp-ni-Pth & „,auserwähltes Abbild des Ptah“ \\
\hline Alexander d. Gr. & T mrin-R ${ }^{\ulcorner} w$ stp.n-Imn.w. $w^{50}$ & „... der, den Amun auserwählt hat“ \\
\hline Philippos Arrhidaios & $\begin{array}{l}\text { T mri-Re } w \text { stp.n-Imn.w. }{ }^{51} \\
\text { T mri-k3-R w stp.n-Imn.w }{ }^{52}\end{array}$ & $\begin{array}{l}\text { „... der, den Amun auserwählt hat"“ } \\
\text { „... der, den Amun auserwählt hat" }\end{array}$ \\
\hline Alexander II. (IV.) & $\mathrm{T} h^{\Upsilon \top}-i b-R^{\top} w$ stp.n-Imn.w & „... der, den Amun auserwählt hat“ \\
\hline
\end{tabular}

\section{ANHANG V}

Namen, die den Schutz Ägyptens explizit aufgreifen:

\begin{tabular}{|c|c|c|}
\hline Taharqa & $\mathrm{G} h w i-t 3 . w i$ & „der die beiden Länder beschützt“ \\
\hline Teos & G hwwi-B $3 k . t w^{\top} f-h 3 s . w t$ & $\begin{array}{l}\text { "der Ägypten beschützt, der die } \\
\text { Fremdländer niederzwingt" }\end{array}$ \\
\hline Nektanebos (II.) & $\mathrm{H}$ mri-t3.wi mki-B $3 k . t$ & $\begin{array}{l}\text { "der die beiden Länder liebt, } \\
\text { der Ägypten beschützt"“ }\end{array}$ \\
\hline Alexander der Große & $\mathrm{H} m k i-K m . t$ & „der Ägypten beschützt“ \\
\hline
\end{tabular}

\section{ANHANG VI}

Namen, die das Schlagen der Feinde thematisieren:

\begin{tabular}{|c|c|c|}
\hline Schebitko & G 3-hpš hwij-pşč.t-pč.t & $\begin{array}{c}\text { "mit großer Schlagkraft, der die Neun } \\
\text { Bogen schlägt" }\end{array}$ \\
\hline Teos & $\mathrm{G} h w i-B 3 k . t w^{e} f-h 3 s . w t$ & $\begin{array}{l}\text { „der Ägypten schützt, der die Fremd- } \\
\text { länder niederzwingt“ }\end{array}$ \\
\hline Nektanebos (II.) & $\begin{array}{l}\text { N s:hrw-ib-nčr.w tkn-h3s.wt } \\
\text { G s:mn-hp.w hwwi-psç.t. }-p c \grave{.} . t\end{array}$ & $\begin{array}{c}\text { "der das Herz der Götter zufriedenstellt, } \\
\text { der den Fremdländern nahekommt" } \\
\text { "der die Gesetze bestehen läßt, der die } \\
\text { Neun Bogen schlägt“" }\end{array}$ \\
\hline Alexander der Große & $\mathrm{H} h k 3-k n i t k n-h 3 s . w t$ & $\begin{array}{l}\text { "tapferer Herrscher, der den Fremd- } \\
\text { ländern nahekommt" }\end{array}$ \\
\hline Philippos Arrhidaios & $\mathrm{N} h k 3-h 3 s . w t$ & „Herrscher der Fremdländer“ \\
\hline
\end{tabular}

${ }^{50}$ Mit De Meulenaere, CRIPEL 13, 1991, 57.

${ }^{51}$ Mit De Meulenaere, CRIPEL 13, 1991, 57.

${ }^{52}$ Mit De Meulenaere, CRIPEL 13, 1991, 54. 
SUMMARY

Names were an important means for the legitimization of Egyptian kings. The Late Period (Dyn. 25 - Alexander II. [IV.]) displays a frequent use of names which were known from former kings, alluded to the protection of the frontiers or the smiting of the enemies, or stressed the king's divine descent. It can be shown that usurpers and foreign rulers had a particular preference for these kinds of names. 